\title{
Aprender a aprender: competencia básica en el marco europeo de la educación obligatoria
}

\section{Learning to learn: basic competence in the European framework of compulsory education}

\author{
Santiago García Vázquez \\ Investigador en Formación y Perfeccionamiento del Departamento de Didáctica y Organización Escolar de la Facultad de Ciencias \\ de la Educación de la Universidad de Santiago de Compostela (España)
}

\begin{abstract}
Resumen
En este trabajo se presenta una aproximación al marco teórico de referencia establecido por el Parlamento Europeo y Consejo de la Unión Europea sobre la competencia aprender a aprender como competencia clave para el aprendizaje permanente en la enseñanza obligatoria. De este modo, se toma como base la realización de un análisis pormenorizado de la delimitación de la competencia aprender a aprender, y el estudio de los factores y aspectos implícitos que conlleva dicha competencia en el marco europeo y su extrapolación a la normativa educativa española (Ley Orgánica de Educación y Ley de Mejora de la Calidad Educativa).

Palabras clave: aprender a aprender, competencias clave, sociedad del conocimiento.
\end{abstract}

\begin{abstract}
This academic work introduce an approximation to the theoretical framework established by the European Parliament and Council of the European Union on the compete learning to learn as a key competence for lifelong learning in compulsory education. Thus, the basis is conducting a detailed analysis of the delimitation of the competence learning to learn, and study of the factors and implicit aspects this competence involved in the European framework and its extrapolation to the Spanish educational standards (Organic Law of Education, and Law on the Improvement of the Educational Quality).

Keywords: learning to learn, key skills and knowledge society.
\end{abstract}

\section{Introducción}

El momento actual de la sociedad, cambiante y globalizada que en las dos últimas décadas se está a experimentar a nivel mundial, cada vez, con mayor intensidad, condiciona notoriamente la preocupación e investigación de los profesionales del ámbito educativo por el diseño de metodologías de aprendizaje innovadoras y el desarrollo eficaz de las competencias clave que los futuros individuos de la sociedad del siglo
XXI deben poseer y les serán demandadas (OCDE, 2002).

Así pues, siguiendo a Az-Zarnuji (1991) quien alude a que la búsqueda del saber no se puede realizar sin seis cuestiones fundamentales: agudeza ingeniosa, deseo ferviente, paciencia, sostenimiento suficiente, la dirección del docente y un período de tiempo bastante largo. Ante ello, cabe inferir que el empeño y la preocupación por la mejora de los procesos de enseñanza-aprendizaje de los discentes, nació con el mismo hecho de la educación. A pesar de que estas palabras datan de la Edad Media, múltiples elementos de la sociedad, la ciencia y la educación han cambiado, como es lógico, en el momento actual que estamos a vivir, pero en ella reside la esencia de investigar y mejorar constantemente el proceso de aprendizaje y su contribución a una enseñanza de calidad y adecuada a las necesidades y demandas sociales (Martín Ortega y Moreno Hernández, 2007, pp. 19-20).

Esta preocupación de mejora de los procesos de enseñanza-aprendizaje, y en particular del proceso de aprendizaje de los discentes, es latente a nivel internacional. Muestra de ello, se plasma en el marco europeo con la Recomendación del Parlamento Europeo y del Consejo, de 18 de diciembre de 2006, sobre las competencias clave para el aprendizaje permanente. Donde en ella, se hace explícito por primera vez en una normativa legislativa educativa la alusión a la competencia aprender a aprender como elemento básico a desarrollar y promocionar en los discentes en su etapa de enseñanza obligatoria.

Esta adecuación de la enseñanza a las necesidades y demandas sociales es reseñada por múltiples autores del ámbito educativo y amparada desde la comunidad científica desde finales del siglo XX y más rotundamente a comienzos del siglo XXI. Ejemplo de ello, es la mención que Suárez-Orozco y Qin-Hillard (2004) hicieron sobre los retos que se le presentan a la educación desde las sociedades constantemente; donde esa importancia por la relevancia del proceso de aprendizaje y su concienciación se vislumbra del 
discurso realizado sobre dicha cuestión y en la que se puede inferir que la flexibilidad cognitiva y la agilidad deben dominar en el aprendizaje, se necesitan personas cognitivamente flexibles, culturalmente sofisticadas y capaces de un trabajo colaborativo en grupos formados por individuos diferentes. Enfoques de enseñanza basados en las multitareas, en el aprender reflexivo y en cómo aprender, en el aprender de los errores, en la relevancia del aprendizaje a lo largo de la vida.

Esta cuestión, es aborda desde el marco europeo de referencia con la Recomendación del Parlamento Europeo y del Consejo, de 18 de diciembre de 2006, sobre las competencias clave para el aprendizaje permanente. Donde en ella, se intenta diseñar un marco común para los estados miembros con la finalidad de responder de manera flexible a las necesidades, demandas y retos de la sociedad actual y cambiante del siglo XXI.

\section{Aprender a aprender en el Marco Europeo}

En la Recomendación del Parlamento Europeo y del Consejo, de 18 de diciembre de 2006, sobre las competencias clave para el aprendizaje permanente. Se hace explícita y con gran detalle alusión al significado de la competencia aprender a aprender junto con las implicaciones que conlleva en el proceso de enseñanzaaprendizaje a lo largo de la enseñanza obligatoria.

En este sentido, la competencia aprender a aprende es la habilidad para iniciar el aprendizaje y persistir en él, para organizar el propio aprendizaje y gestionar el tiempo y la información eficazmente, ya sea de manera individual o grupal. Esta competencia conlleva ser consciente del propio proceso de aprendizaje y de las necesidades de aprendizaje de cada uno, determinando las oportunidades disponibles y siendo capaz de superar los obstáculos con el fin de culminar el aprendizaje exitosamente. Dicha competencia significa adquirir, procesar y asimilar nuevos conocimientos y capacidades, así como buscar orientaciones y hacer uso de ellas. El hecho de aprender a aprender hace que el alumnado se apoye en experiencias vitales y de aprendizajes anteriores con el fin de utilizar y aplicar los nuevos conocimientos y capacidades en muy diversos contextos, como los de la vida privada y profesional, y la educación y formación. La motivación y la confianza son cruciales para la adquisición de esta competencia.

Asimismo, el desarrollo de esta competencia implica una serie de conocimientos, capacidades y actitudes a promover en el alumnado; ellas son:

- Cuando el aprendizaje se dirige a la consecución de un empleo determinado o de objetivos profesionales, la persona debe tener conocimiento de las competencias, los conocimientos, las capacidades y las cualificaciones exigidos. En todos los casos, aprender a aprender exige que la persona conozca y sepa qué estrategias de aprendizaje son sus preferidas, los puntos fuertes y débiles de sus capacidades y cualificaciones, y que sea capaz de buscar las oportunidades de educación y formación y los servicios de apoyo y orientación a los que puede acceder.
- Para empezar, aprender a aprender exige la adquisición de las capacidades básicas fundamentales necesarias para el aprendizaje complementario, como la lectura, la escritura, el cálculo y las Tecnologías de la Información y Comunicación (TIC). A partir de esta base, la persona debe ser capaz de acceder a nuevos conocimientos y capacidades, y de adquirirlos, procesarlos y asimilarlos. Esto exige que la persona gestione eficazmente su aprendizaje, su carrera y su actividad profesional y, en particular, que sea capaz de perseverar en el aprendizaje, de concentrarse en períodos de tiempo prolongados y de reflexionar críticamente sobre los fines y el objeto del aprendizaje. Asimismo, de las personas se espera que sean autónomas y autodisciplinadas en el aprendizaje, pero también que sean capaces de trabajar en equipo, de sacar partido de su participación en un grupo heterogéneo y de compartir lo que hayan aprendido. Las personas han de ser capaces de organizar su propio aprendizaje, de evaluar el trabajo propio y, llegado el caso, de procurarse asesoramiento, información y apoyo.

- Toda actitud positiva debe basarse en la motivación y la confianza para iniciar y culminar con éxito el aprendizaje a lo largo de la vida. La capacidad de las personas de aprender, de superar los obstáculos y de cambiar se sustenta en una actitud positiva orientada a la resolución de problemas. El deseo de aplicar lo aprendido y lo vivido anteriormente, y la curiosidad que impulsa a buscar oportunidades de aprender y aplicar lo aprendido a diversos contextos vitales, son elementos esenciales de una actitud positiva.

De un análisis pormenorizado, puede extraerse que la competencia de aprender a aprender reside en la concienciación por parte del discente del proceso de aprendizaje desarrollado, donde la motivación y confianza son elementos transcendentales en el mismo, junto a otros elementos y estrategias implícitas como búsqueda de información, discriminación de la misma, gestión del tiempo, autodisciplina en el aprendizaje, evaluación del trabajo realizado, superación de metas, entre otros. Cuestiones vinculadas directamente con el proceso de aprender a aprender y que el discente debería ser competente desde edades tempranas para tomar consciencia del proceso de aprendizaje y favorecer el llamado aprendizaje a lo largo de toda la vida; lo que supondría, poseer un mayor dominio y autonomía a la hora de afrontar retos y metas, máxime de cara a la realización de su actividad educativa, profesional y personal.

\section{Aprender a aprender en el Marco Estatal de España}

En cuanto al Marco Estatal de España sobre la legislación educativa referente a la competencia aprender a aprender, cabe citar la recogida en la Ley Orgánica 2/2006, de 3 de mayo, de Educación (LOE), derogada por la Ley Orgánica 8/2013, de 9 de diciembre, para la Mejora de la Calidad Educativa (LOMCE). En ella, se tienen presentes las recomendaciones propuestas desde el Marco Europeo de 
referencia sobre las competencias clave para el aprendizaje permanente. Así pues, desde esta perspectiva la competencia aprender a aprender supone disponer de habilidades para iniciarse en el aprendizaje y ser capaz de continuar aprendiendo de manera cada vez más eficaz y autónoma de acuerdo a los propios objetivos y necesidades.

Esta competencia posee dos dimensiones fundamentales. Por un lado, la adquisición de la conciencia de las propias capacidades (intelectuales, emocionales, físicas), del proceso y las estrategias necesarias para desarrollarlas, así como de lo que se puede hacer por uno mismo y de lo que se puede hacer con ayuda de otras personas o recursos. Y, por otro lado, implica disponer de un sentimiento de competencia personal, que redunda en la motivación, la confianza en uno mismo y el gusto por aprender.

Significa ser consciente de lo que se sabe y de lo que es necesario aprender, de cómo se aprende, y de cómo se gestionan y controlan de forma eficaz los procesos de aprendizaje, optimizándolos y orientándolos a satisfacer objetivos personales. Requiere conocer las propias potencialidades y carencias, sacando provecho de las primeras, y teniendo motivación y voluntad para superar las segundas desde una expectativa de éxito, aumentando progresivamente la seguridad para afrontar nuevos retos de aprendizaje.

Por ello, comporta tener conciencia de aquellas capacidades que entran en juego en el aprendizaje, como la atención, la concentración, la memoria, la comprensión y la expresión lingüística o la motivación de logro, entre otras, y obtener un rendimiento máximo y personalizado de las mismas con la ayuda de distintas estrategias y técnicas: de estudio, de observación y registro sistemático de hechos y relaciones, de trabajo cooperativo y por proyectos, de resolución de problemas, de planificación $\mathrm{y}$ organización de actividades y tiempos de forma efectiva, o del conocimiento sobre los diferentes recursos y fuentes para la recogida, selección y tratamiento de la información, incluidos los recursos tecnológicos.

Implica asimismo la curiosidad de plantearse preguntas, identificar y manejar la diversidad de respuestas posibles ante una misma situación o problema utilizando diversas estrategias y metodologías que permitan afrontar la toma de decisiones, racional y críticamente, con la información disponible. Incluye, además, habilidades para obtener información (ya sea individualmente $\mathrm{o}$ en colaboración) $\mathrm{y}$, muy especialmente, para transformarla en conocimiento propio, relacionando e integrando la nueva información con los conocimientos previos y con la propia experiencia personal y sabiendo aplicar los nuevos conocimientos y capacidades en situaciones parecidas y contextos diversos.

Por otra parte, esta competencia requiere plantearse metas alcanzables a corto, medio y largo plazo y cumplirlas, elevando los objetivos de aprendizaje de forma progresiva y realista.

Hace necesaria, también, la perseverancia en el aprendizaje, desde su valoración como un elemento que enriquece la vida personal y social, y que es, por tanto, merecedor del esfuerzo que requiere. Conlleva ser capaz de autoevaluarse y autorregularse, responsabilidad y compromiso personal, saber administrar el esfuerzo, aceptar los errores y aprender de y con los demás.

En síntesis, la competencia aprender a aprender implica la conciencia, gestión y control de las propias capacidades y conocimientos en el discente desde un sentimiento de competencia o eficacia personal, e incluye tanto el pensamiento estratégico, como la capacidad de cooperar, de autoevaluarse, y el manejo eficiente de un conjunto de recursos y técnicas de trabajo intelectual; todo lo cual, se desarrolla a través de experiencias de aprendizaje conscientes y gratificantes, tanto individuales como colectivas.

\section{Consideraciones finales}

Como consideraciones finales de un análisis pormenorizado de la competencia aprender a aprender desde el marco legislativo vigente a nivel europeo y estatal, y siguiendo a (Martín Ortega y Moreno Hernández, 2007), cabe señar que:

- Aprender a aprender enfatiza en la consciencia de los discentes del propio proceso de aprendizaje, donde los elementos fundamentales para el desarrollo de dicha competencia son la motivación y confianza del alumnado en el proceso de búsqueda de información en diversas fuentes, la elaboración de su posicionamiento al respecto y la comunicación de su perspectiva a las demás personas teniendo presentes los diferentes códigos o lenguajes para ello.

- Asimismo, en la promoción de la competencia aprender a aprender, se alude a factores como confianza, reflexión, auto-descubrimiento, autonomía, aceptación de uno mismo, etc. Aspectos ligados a la configuración de la propia identidad y desarrollo de la autonomía del discente; pero con especial atención a la toma de conciencia y responsabilidad de ello. Pues esa concienciación es la clave fundamental para promover y desarrollar los aprendizajes y resolución de problemas en las etapas futuras y el aprendizaje a lo largo de la vida.

- De un análisis de las materias que configuran el currículum de la enseñanza obligatoria se constata la existencia de materias (Lengua Materna, Lengua Extranjera, Matemáticas) que poseen más explícitamente las implicaciones que supone la competencia aprender a aprender. Pero ante ello, no debe caerse en una visión reduccionista del aprender a aprender, sino abordar dicha competencia y fomentarla de manera transversal en la totalidad de materias que configuran el currículum.

- Aprender a aprender, también, implica en los estudiantes la concienciación de la existencia de diversas perspectivas sobre una misma realidad. Ahí es donde reside el respeto por todas ellas y la discriminación a favor de la más adecuada a juicio propio de los valores sociales y democráticos.

- En el aprender a aprender, son múltiples los elementos que en las normativas legislativas se citan como potenciadores de esta competencia a tener 
presentes por el profesorado de cara al proceso de enseñanza-aprendizaje, tanto cognitivos, afectivos y sociales. Así pues, dichos elementos centrales han de tenerse presente para el desarrollo y promoción de la competencia en los discentes, junto a prácticas innovadoras que potencien esa dimensión del metaaprendizaje.

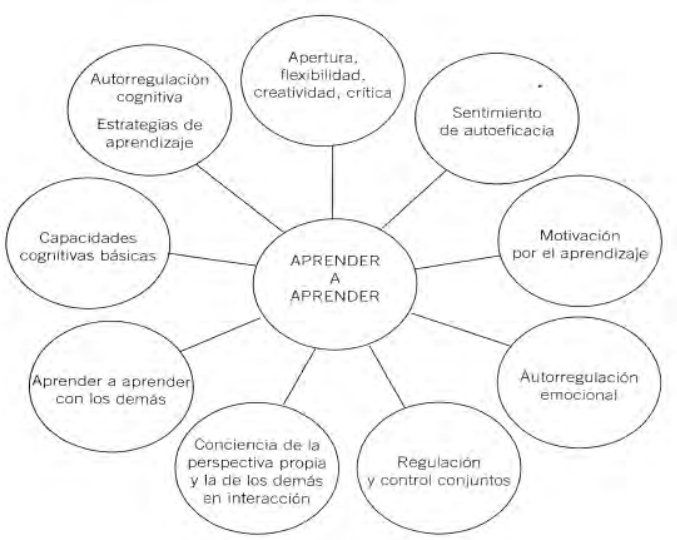

Figura 1. Elementos cognitivos, afectivos y sociales en la competencia aprender a aprender.

Fuente: Martín Ortega y Moreno Hernández, 2007.

- Aprender a aprender, conlleva tener consciencia de las limitaciones y potencialidades propias; pero a su vez, ser conocedor de las diversas estrategias disponibles de cara a la resolución de problemas o el logro de metas. En este sentido, el discente debe ser consciente de ellos y saber qué estrategias son más adecuadas en función de las actividades propuestas, el trabajo a realizar o en función de los rasgos propios.

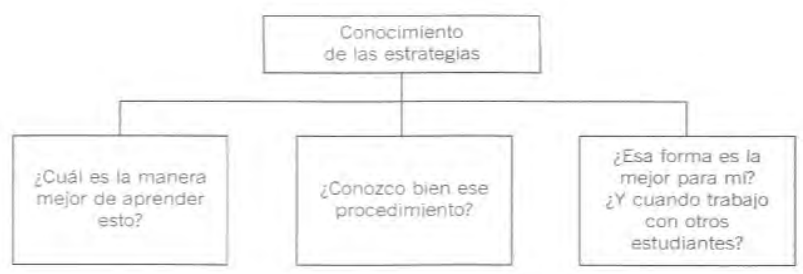

Figura 2. Consciencia sobre el conocimiento de las estrategias en el aprender a aprender.

Fuente: Martín Ortega y Moreno Hernández, 2007.

Como síntesis final, es preciso señalar que la competencia aprender a aprender implica múltiples elementos y factores en su desarrollo y promoción de cara al alumnado (motivación, confianza en si mismo, autorregulación, conciencia de las capacidades propias, gestión y control de tiempos, búsqueda y discriminación de información en diversas fuentes, pensamiento estratégico, capacidad de cooperación, recursos y técnicas de trabajo intelectual, aprendizaje grupal, entre otras). Pero, a pesar de las pautas normativas delimitadas en la legislación educativa europea y estatal española, la clave fundamental reside en el trabajo realizado en las propias aulas de los centros educativos. Pues ahí, se produce el tránsito de lo normativo a la praxis educativa y ello va a estar condicionado por la interpretación del currículum por parte de los docentes, la incorporación de dichas recomendaciones a sus programaciones y la metodología desarrollada en el proceso de enseñanza-aprendizaje.

No obstante, ante este reto nada simple al que se enfrentan los docentes y con la intencionalidad de dejar abierto el debate y la indagación futura sobre esta compleja cuestión presentada; cabría reflexionar y analizar pormenorizadamente sobre la formación inicial y permanente del profesorado al respecto del enfoque de la enseñanza por competencias, el desarrollo de estrategias para promover y fomentar del aprender a aprender, técnicas de estudio, aprendizaje significativo y autónomo, o actividades para promocionar los procesos metacognitivos, razonamiento y debate.

\section{Referencias}

Az-Zarnuji (1991). Instrucción del estudiante. El método de aprender. Madrid: Hiparión.

Garton, A. (2004). Exploring cognitive development. Oxford: Blackwell.

Lacasa, P. y Herranz, P. (1995). Aprendiendo a aprender. Resolver problemas entre iguales. Madrid: CIDE.

Organización para la Cooperación y el Desarrollo Económicos (OCDE) (2002). Definition and Selection of Competencies (DeSeCo): Theoretical and Conceptual Foundations. París: OCDE.

Martín Ortega, E. y Moreno Hernández, A. (2007). Competencia para aprender a aprender. Madrid: Alianza.

Ministerio de Educación, Cultura y Deporte (MECD) (2013). Ley Orgánica 8/2013, de 9 de diciembre, para la Mejora de la Calidad Educativa. Boletín Oficial del Estado $\mathrm{n}^{\mathrm{o}} 295$, de 10 de diciembre de 2013. Madrid: MECD. http://www.boe.es/boe/dias/2013/ 12/10/pdfs/BOE-A-2013-12886.pdf

Ministerio de Educación y Ciencia (MEC) (2006). Ley Orgánica, 2/2006, de 3 de mayo, de Educación. Boletín Oficial del Estado $\mathrm{n}^{\mathrm{o}}$ 106, de 4 de mayo de 2006. Madrid: MEC. http://www.boe.es/boe/dias/ 2006/05/04/pdfs/A17158-17207.pdf

Sanmartí, N. (2007). Evaluar para aprender. 10 ideas claves. Barceló: Graó

Suárez-Orozco, M. M. y Quin-Hilliard, D. B. (2004). Globalization. Culture and Education in the New Millennium. California: The University of California Press.

Unión Europea (2006). Recomendación del Parlamento Europeo y del Consejo, de 18 de diciembre de 2006, sobre las competencias clave para el aprendizaje permanente. Diario Oficial de la Unión Europea (2006/962/CE). Bruselas: Comisión Europea.

http://www.mcu.es/cine/docs/Novedades/Recomendac ion_Parlamento_Europeo_Consejo_Aprendizaje_per manente.pdf

\section{Agradecimientos}

Agradecer a la Xunta de Galicia la viabilidad de la presente investigación por su financiamiento bajo la subvención de la Red de Investigación TELGalicia R2014/029. 